\title{
Mesh size and bird capture rates in shasha forest reserve, ile-ife, Nigeria
}

\author{
Akinsola I. Akinpelu \\ Department of Zoology, Obafemi Awolowo University, Ile-lfe, Nigeria \\ e-mail: sholaakinpelu@yahoo.com
}

\begin{abstract}
Few studies have been conducted to verify how mist net mesh size affects the capture rates of birds in Nigeria. This research was undertaken from 06.30 to 18.30 hours between February 2010 and January 2011 at Shasha Forest Reserve $\left(4^{\circ} 20^{\prime}\right.$ to $4^{\circ} 40^{\prime} \mathrm{E}, 7^{\circ} 00^{\prime}$ to $\left.7^{\circ} 10^{\prime} \mathrm{N}\right)$ near lle-lfe in southwestern Nigeria, to verify how mesh size affects the capture rates of birds and the efficiency or otherwise of the mist nets involved. Bird species occurrence was recorded using eight nylon mist nets each of $36 \mathrm{~mm}$ and $61 \mathrm{~mm}$ mesh sizes in linear transects. The linear transects cuts across different vegetational types with varying degrees of human disturbance. A total of 970 birds were captured and made up of $584(60.2 \%)$ with $36 \mathrm{~mm}$ mesh and $386(39.8 \%)$ with $61 \mathrm{~mm}$ mesh. Of the 106 species mist-netted $93(87.7 \%)$ were caught by $36 \mathrm{~mm}$ while $61 \mathrm{~mm}$ caught $73(68.9 \%)$. The $36 \mathrm{~mm}$ mesh net was more effective for birds with less than $200 \mathrm{~mm}$ body length while $61 \mathrm{~mm}$ mesh was more effective for birds with more than $250 \mathrm{~mm}$ body length. However, both meshes were suitable for birds between 151 and $200 \mathrm{~mm}$ long. Thirty six millimeter mesh net captured most of the birds below $20 \mathrm{~g}$ while $61 \mathrm{~mm}$ mesh captured was more efficient for the larger species. Both mesh sizes were effective for birds with body weights between 41.00 and $80.00 \mathrm{~g}$. Deployment of both nets will provide an improvement of abundance estimates for some larger species. By using only $36 \mathrm{~mm}$ mesh nets, there may be an improvement in the capture rate of small birds and similarly $61 \mathrm{~mm}$ mesh would substantially improve the capture rate of bigger and heavier birds.
\end{abstract}

Key words: Mesh size, birds, capture rates, body weight, Shasha reserve.

\section{INTRODUCTION}

Mist nets have been used in many studies of bird populations and their results compared with other methods [1-3]. However, since far more can be learned from netting studies, a simple theory of net capture rates has been employed. Although Rappole et al.[4] have proposed a methodology that combines two procedures, i.e., mist nets and point counts, to produce a more accurate assessment of avian habitat use, the use of mist nets is still considered as the best assessment method to date. According to Poulin et al., [3], while point counts are less demanding in the field, the manipulation of netted birds allows documentation of various aspects of their biology. However, at least two factors, according to Macarthur and Macarthur [1], must be recognized in any use of mist for population estimates. First, when a bird has once been captured, marked, and released, it tends to avoid nets in the future. This tendency is more pronounced in the tropics than in the temperate regions. Hence, traditional capture - recapture methods to estimate populations cannot be used. Secondly, the population is composed of two or more parts, i.e. territorial or resident species in which the birds are continually exposed to the likelihood of capture and are almost all eventually caught, marked and released. The other aspect consists of drifting birds that seem to flow past the nest site at almost constant rate, bringing in almost everyday, new previously unmarked birds, irrespective of the number of days the nets have been in operation. Apart from this, a lot of other restrictions concerning mist-net use were listed by Remsen and Good [5].

It is not yet known how to measure with complete accuracy all the factors involved in a birds' being caught and held in a net. Such measurements as body weight, total length, width of skull and length of wings, legs, toes and claws can be taken easily but it is much more difficult to measure the stiffness of feathers and shape of the head, body, wings, legs, tail, etc.. The velocity of the bird when it hits the net and its condition (wet or dry), and the weather especially the wind. In view of this complexity, a convenient index of body size, i.e., total length of the body and body weight are chosen.

However, when mist nets are employed, the question arises as to which mesh size produces more captures per hour, since why a species cannot be sampled is its size, i.e., it can either be too small or too big to get entangled in the nets [1]. Jenni et al. [6], while working on capture rate efficiency of 36 mm-mesh concluded that different species and 
climatic condition can provide different capture rates for $36 \mathrm{~mm}$ nets. In most works involving mist net captures, mesh sizes are not mentioned for Costa Rica birds [7]; for Californian birds [8] and for Brazilian birds [9].

This study presents the profiles and comparison of bird capture efficiency of mist nets with two mesh sizes (36 and $61 \mathrm{~mm}$ ) in Shasha forest reserve located in the rainforest belt of South Western Nigeria.

\section{MATERIALS AND METHODS}

Eight nylon mist nets each of 36- and 61-mm were alternately spaced for twelve months (February 2010 to January 2011) in linear transects. All the nets had the same dimension $(2 \times 2.6 \mathrm{~m})$ and were operated from 06.30 to 18.30 hours in four locations. The study plots in Shasha forest reserve $\left(4^{\circ} 20^{\prime}\right.$ to $4^{\circ} 40^{\prime} \mathrm{E}, 7^{\circ} 00^{\prime}$ to $7^{\circ} 10^{\prime} \mathrm{N}$ ) were located in the tropical humid high forest coupled with a forest formation resulting from disturbance of the primary forest by farming and logging. The study plots were characterized by the presence of evergreen tree species of economic importance like the oil palm (Elaeis guineensis) the mahogany (Khaya ivorensis and Khaya grandifiola), Sapele wood (Entadrophragma cylindricum), iroko (Milicia excelsa), African walnut (Lovoa klaineana), Obeche (Triplochiton scleroxylon), Opepe (Sacrocephalus diderrichii), afara (Terminalia superba), among others that are of increasing commercial importance. The four study sites sampled four different vegetation types, with varying degrees of human disturbance, i.e. primary forest, closed-canopy tall trees, gallery forest and degraded secondary regrowth forest. Although sampling effort varied between study sites, both 36-mm and 61-mm mesh nets were always simultaneously used in equal numbers. Each bird handled, that was removed from the experimental nets, was recorded by species and mesh size in which it had been caught. At the end of the period, the number of each species was tallied for the two mesh sizes. For alternating 36- and 61-mm mesh nets, captures relative to bird length measured by means of a metal ruler and body weight measured with 50-, 100-, 300- and $1000 \mathrm{~g}$ spring scales were determined. $\mathrm{Chi}^{2}$ tests were used to check statistical significance of different capture rates and Yate's correction for continuity was used when there were only two categories in the distribution [10].

The birds were identified as previously described [11].

\section{RESULTS}

During the 4380 net hours, 970 birds were mistnetted, made up of $584(60.2 \%)$ with $36 \mathrm{~mm}$ and 386 $(39.8 \%)$ with $61 \mathrm{~mm}$. Of the 106 mist-netted species, $93(87.7 \%)$ were caught by $36 \mathrm{~mm}$ while $61 \mathrm{~mm}$ mesh caught $73(68.9 \%)$. Because of the large number of species in the series, analysis of data by body length and weight became meaningful.

Table 1 shows that $36 \mathrm{~mm}$ mesh nets captured most of the bird species below $20 \mathrm{~g}$ (Alcedo quadribrachys, Muscicapa caerulescens, Nectarinia superba, Nectarinia olivacea and Nigrita luteifrons). However, for some larger species, $61 \mathrm{~mm}$ mesh size was more efficient. This is the situation with Streptopelia semitorquata, Treron australis, Tockus hartlaubi and Campethera nivosa.

For 19 species, differences in capture rates were statistically significant $(P<0.05)$ and 16 species (Apus apus, Alcedo quadribrachys, Eurystomus gularis, Eurystomus glaucurus, Phoeniculus bollei, Campethera nivosa, Campethera chloronota, Hylia prasina, Muscicapa caerulescens, Anthreptes collaris, Nectarinia cyanolaema, Nectarinia olivacea, Nectarinia superba, Nigrita bicolor, Nigrita fusconota and Nigrita luteifrons) were caught more with $36 \mathrm{~mm}$ mesh size while only three species (Treron australis, Pitta angolensis and Malaconotus cruentus) were captured more with $61 \mathrm{~mm}$ mesh size.

The $36 \mathrm{~mm}$ mesh nets captured birds from 48.00 to $551.00 \mathrm{~mm}$ long with a mean of $191.29 \pm 8.71 \mathrm{~mm}$, while the $61 \mathrm{~mm}$ mesh captured birds from 51 to $770 \mathrm{~mm}$ long with a mean of $226.36 \pm 15.40 \mathrm{~mm}$. Both meshes were more efficient for birds with body length between 151 and $200 \mathrm{~mm}$ (Fig. 1).

Body weights of birds captured with $36 \mathrm{~mm}$ mesh ranged from 5.00 to $366.00 \mathrm{~g}$ with a mean of $54.69 \pm 7.80 \mathrm{~g}$, while those captured with $61 \mathrm{~mm}$ mesh ranged from 8.00 to $921.00 \mathrm{~g}$ with a mean of $104.00 \pm 18.09 \mathrm{~g}$. Both mesh sizes were effective for birds with body weights between 41.00 and $80.00 \mathrm{~g}$ even though $36 \mathrm{~mm}$ mesh captured more birds. For birds between 81.00 and $120.00 \mathrm{~g}$ both meshes captured almost similar number but beyond this weight range $61 \mathrm{~mm}$ mesh was more efficient (Fig. 2).

Table 2 displays the mean number of observed but unmist-netted birds within the study area. Twelve species belonging to 6 families were recorded and they were found to be aerial in activity. 
Table 1: Systematic list of bird species and number of captures (without recaptures) mist-netted in Shasha Forest Reserve, lle-lfe, with 36- and 61-mm mesh, with mean body weight (g) and mean total length (mm)

\begin{tabular}{|c|c|c|c|c|c|c|}
\hline \multirow[b]{2}{*}{ Common name } & \multirow[b]{2}{*}{ Family/species } & \multicolumn{2}{|c|}{ Mean } & \multicolumn{2}{|c|}{ Mist net mesh size } & \multirow[b]{2}{*}{$\begin{array}{c}\text { Total } \\
\text { captures }\end{array}$} \\
\hline & & $\begin{array}{c}\text { Mean } \\
\text { weight }(\mathrm{g})\end{array}$ & $\begin{array}{l}\text { Total length } \\
(\mathrm{mm})\end{array}$ & $36 \mathrm{~mm}$ & $61 \mathrm{~mm}$ & \\
\hline & Phasianidae & & & & & \\
\hline Latham's Francolin & Francolinus lathami & 254.5 & 228.6 & 0 & 5 & 5 \\
\hline Ahanta Francolin & $\begin{array}{l}\text { Francolinus ahantensis } \\
\text { Columbidae }\end{array}$ & 510.1 & 314.8 & 0 & 2 & 2 \\
\hline Grey Wood Pigeon & Columba unicincta & 423.4 & 406.4 & 0 & 5 & 5 \\
\hline Red-eyed Dove & Streptopelia semitorquata & 196.2 & 330.2 & 6 & 11 & 17 \\
\hline Green Fruit-pigeon & Treron australis & 210.3 & 279.4 & 3 & 16 & 19 \\
\hline Blue-headed Dove & Turtur brehmeri & 133.1 & 254.0 & 1 & 4 & 5 \\
\hline Tambourine Dove & $\begin{array}{l}\text { Turtur tympanistria } \\
\text { Musophagidae }\end{array}$ & 196.3 & 330.2 & 2 & 9 & 11 \\
\hline Blue Plaintain-eater & Corythaeola cristata & 903.0 & 762.0 & 0 & 1 & 1 \\
\hline Verreaux's Tauraco & Tauraco macrorhynchus & 246.0 & 431.8 & 0 & 2 & 2 \\
\hline Green-Crested Tauraco & $\begin{array}{l}\text { Tauraco persa } \\
\text { Cuculidae }\end{array}$ & 248.4 & 431.6 & 1 & 2 & 3 \\
\hline Black-throated Coucal & Centropus leucogaster & 293.0 & 355.6 & 1 & 5 & 6 \\
\hline Senegal Coucal & Centropus senegalensis & 291.0 & 355.6 & 0 & 2 & 2 \\
\hline Didric Cuckoo & Chrysococcyx caprius & 36.7 & 190.5 & 18 & 13 & 31 \\
\hline Emerald Cuckoo & Chrysococcyx cupreus & 38.2 & 228.6 & 4 & 0 & 4 \\
\hline Black Cuckoo & $\begin{array}{l}\text { Cuculus clamosus } \\
\text { Caprimulgidae }\end{array}$ & 82.0 & 304.8 & 2 & 3 & 5 \\
\hline Black-shouldered Nightjar & $\begin{array}{l}\text { Caprimulgus pectroralis } \\
\text { Apodidae }\end{array}$ & 45.6 & 228.6 & 1 & 1 & 2 \\
\hline European Swift. & Apus apus & 41.8 & 167.1 & 9 & 1 & 10 \\
\hline Cassin's spine-tailed Swift & Chaetura cassini & 51.0 & 152.4 & 0 & 2 & 2 \\
\hline Ussher's spine-tailed Swift & $\begin{array}{l}\text { Chaetura ussheri } \\
\text { Trogonidae }\end{array}$ & 55.0 & 139.7 & 0 & 1 & 1 \\
\hline Narina Trogon & $\begin{array}{l}\text { Apaloderma narina } \\
\text { Alcedinidae }\end{array}$ & 62.3 & 304.8 & 0 & 1 & 1 \\
\hline Shining-blue kingfisher & Alcedo quadribrachys & 15.0 & 190.5 & 21 & 3 & 24 \\
\hline Red-headed Dwarf Kingfisher & Ceyx lecontei & 10.5 & 101.6 & 6 & 1 & 7 \\
\hline Pigmy Kingfisher & Ceyx picta & 10.0 & 139.7 & 2 & 0 & 2 \\
\hline Chocolate-backed Kingfisher & $\begin{array}{l}\text { Halcyon badia } \\
\text { Meropidae }\end{array}$ & 52.6 & 203.2 & 8 & 2 & 10 \\
\hline Blue-headed Bee-eater & $\begin{array}{l}\text { Merops muelleri } \\
\text { Coracidae }\end{array}$ & 28.3 & 152.4 & 5 & 5 & 10 \\
\hline Broad-billed Roller & Eurystomus glaucurus & 14.8 & 254.0 & 6 & 0 & 6 \\
\hline Blue-throated Roller & $\begin{array}{l}\text { Eurystomus gularis } \\
\text { Upupidae }\end{array}$ & 16.4 & 254.3 & 7 & 1 & 8 \\
\hline Buff-headed Wood-Hoopoe & $\begin{array}{l}\text { Phoeniculus bollei } \\
\text { Bucerotidae }\end{array}$ & 66.5 & 330.2 & 11 & 3 & 14 \\
\hline Blue-billed Dwarf Hornbill & Tockus camurus & 112.6 & 381.0 & 2 & 9 & 11 \\
\hline Black and white tailed Hornbill & Tockus fasciatus & 278.0 & 533.4 & 2 & 7 & 9 \\
\hline Black Dwarf Hornbill & Tockus hartlaubi & 118.0 & 381.0 & 16 & 22 & 38 \\
\hline White-crested Hornbill & $\begin{array}{l}\text { Tropicranus albocristatus } \\
\text { Capitonidae }\end{array}$ & 297.0 & 762.0 & 0 & 3 & 3 \\
\hline Naked-faced Barbet & Gymnobucco calvus & 50.1 & 203.2 & 5 & 9 & 14 \\
\hline Bristle-mosed Barbet & Gymnobucco peli & 52.0 & 201.8 & 6 & 11 & 17 \\
\hline Red-rumped Tinker-bird & Pogoniulus atro-flavus & 18.0 & 181.9 & 2 & 0 & 2 \\
\hline Lemon-rumped Tinker-bird & Pogoniulus bilineatus & 13.1 & 188.8 & 1 & 1 & 2 \\
\hline Speckled Tinker-bird & Pogoniulus scolopaceus & 16.3 & 190.5 & 6 & 2 & 8 \\
\hline Yellow-throated Tinker-bird & Pogoniulus subsuiphureus & 47.1 & 191.1 & 4 & 5 & 9 \\
\hline Yellow-billed Barbet & Trachyphonus purpuratus & 86.5 & 193.3 & 3 & 1 & 4 \\
\hline
\end{tabular}




\begin{tabular}{|c|c|c|c|c|c|c|}
\hline & Indicatoridae & & & & & \\
\hline Lyre-tailed Honey-guide & Melichneutes robustus & 600.1 & 246.7 & 0 & 1 & 1 \\
\hline \multirow{2}{*}{$\begin{array}{l}\text { Cassin's Sharp-billed Honey- } \\
\text { guide }\end{array}$} & Prodotiscus insignis & 38.6 & 177.8 & 1 & 1 & 2 \\
\hline & Picidae & & & & & \\
\hline Brown-eared Woodpecker & Campethera caroli & 322.1 & 175.2 & 31 & 41 & 72 \\
\hline Buff-spotted Woodpecker & Campethera nivosa & 42.5 & 152.4 & 17 & 0 & 17 \\
\hline Fire-bellied Woodpecker & Mesopicos pyrrhogaster & 70.0 & 244.3 & 34 & 25 & 59 \\
\hline Pigmy Woodpecker & $\begin{array}{l}\text { Verreauxia africana } \\
\text { Eurylamidae }\end{array}$ & 5.9 & 76.2 & 1 & 0 & 1 \\
\hline Rufous-sided Broadbill & $\begin{array}{l}\text { Smithornis rufolateralis } \\
\text { Pittidae }\end{array}$ & 29.3 & 127.0 & 13 & 6 & 19 \\
\hline Angola Pitta & $\begin{array}{l}\text { Pitta angolensis } \\
\text { Laniidae }\end{array}$ & 36.4 & 174.8 & 3 & 11 & 14 \\
\hline $\begin{array}{l}\text { Black-shouldered Puff-back } \\
\text { shrike }\end{array}$ & Dryoscopus senegalensis & 11.6 & 165.1 & 2 & 0 & 2 \\
\hline Fiery-breasted Bush-shrike & Malaconotus cruentus & 70.2 & 251.4 & 3 & 14 & 17 \\
\hline Many-coloured Bush-shrike & Malaconotus multicolor & 53.2 & 228.6 & 1 & 0 & 1 \\
\hline Red-billed shrike & $\begin{array}{l}\text { Prionops caniceps } \\
\text { Oriolidae }\end{array}$ & 51.1 & 203.2 & 4 & 8 & 12 \\
\hline Black-headed Oriole & Oriolus brachyrhynchus & 46.4 & 254.2 & 4 & 1 & 5 \\
\hline Black-winged Oriole & $\begin{array}{l}\text { Oriolus nigripennis } \\
\text { Dicruridae }\end{array}$ & 38.7 & 236.7 & 3 & 1 & 4 \\
\hline Shining Drongo & $\begin{array}{l}\text { Dicrurus atripennis } \\
\text { Campephagidae }\end{array}$ & 48.3 & 227.6 & 1 & 0 & 1 \\
\hline Blue Cuckoo-shrike & $\begin{array}{l}\text { Coracina azurea } \\
\text { Covidae }\end{array}$ & 22.0 & 215.9 & 2 & 1 & 3 \\
\hline Bare-headed Rock-fowl & $\begin{array}{l}\text { Picathartes gymnocephalus } \\
\text { Pycnonotidae }\end{array}$ & 355.6 & 431.8 & 1 & 2 & 3 \\
\hline Little Grey Bulbul & Andropadus gracilis & 21.6 & 167.8 & 21 & 14 & 35 \\
\hline Yellow-whiskered bulbul & Andropadus latirostris & 25.2 & 152.4 & 6 & 2 & 8 \\
\hline Little Green Bulbul & Andropadus virens & 24.7 & 155.1 & 1 & 0 & 1 \\
\hline Honey-guide Bulbul & Baeopogon indicator & 48.1 & 271.4 & 4 & 5 & 9 \\
\hline Green-tailed Bristle-bill & Bleda eximia & 48.3 & 52.7 & 9 & 9 & 18 \\
\hline Bristle-bill & Bleda syndactyla & 46.0 & 173.6 & 4 & 5 & 9 \\
\hline Bearded Bulbul & Criniger barbatus & 44.4 & 167.2 & 1 & 1 & 2 \\
\hline White-bearded Bulbul & Criniger calurus & 32.1 & 127.8 & 3 & 1 & 4 \\
\hline West African Nicator & Nicator chloris & 38.2 & 164.8 & 3 & 0 & 3 \\
\hline Lesser icterine Greenbul & $\begin{array}{l}\text { Phyllastrephus icterinus } \\
\text { Turdidae }\end{array}$ & 20.8 & 165.1 & 21 & 16 & 37 \\
\hline Fire-crest Alethe & Alethe diademata & 32.0 & 203.2 & 6 & 1 & 7 \\
\hline Forest Scrub-robin & Cercotrichas leucosticta & 24.0 & 165.1 & 1 & 0 & 1 \\
\hline White-tailed Ant-thrush & Neocossyhus poensis & 57.1 & 214.9 & 2 & 3 & 5 \\
\hline Forest Robin & Stiphrornis erythrothorax & 15.2 & 127.0 & 11 & 1 & 12 \\
\hline Fraser's Rusty Thrush & $\begin{array}{l}\text { Stizorhina frazeri } \\
\text { Timalidae }\end{array}$ & 38.7 & 201.1 & 1 & 2 & 3 \\
\hline Capuchin Babbler & Phyllanthus atripennis & 28.0 & 241.3 & 6 & 8 & 14 \\
\hline Brown Akalat & $\begin{array}{l}\text { Malacocincla fulvescens } \\
\text { Sylviidae }\end{array}$ & 37.1 & 215.8 & 2 & 0 & 2 \\
\hline Stream Warbler & $\begin{array}{l}\text { Bathmocercus } \\
\text { cerviniventris }\end{array}$ & 16.4 & 127.0 & 2 & 0 & 2 \\
\hline Green-backed Camaroptera & Camaroptera chloronota & 11.2 & 114.3 & 10 & 3 & 13 \\
\hline Yellow-browed Camaroptera & Camaroptera superciliaris & 8.1 & 111.2 & 8 & 4 & 12 \\
\hline Green Hylia & Hylia prasina & 12.1 & 114.2 & 7 & 0 & 7 \\
\hline Olive Longbill & Macrosphenus concolor & 14.3 & 118.1 & 1 & 0 & 1 \\
\hline Tit-Hylia & Pholidornis rushiae & 13.8 & 76.2 & 4 & 0 & 4 \\
\hline
\end{tabular}




\begin{tabular}{|c|c|c|c|c|c|c|}
\hline & Muscicapidae & & & & & \\
\hline Dusky Flycatcher & Artomyias fulginosa & 11.6 & 121.4 & 8 & 4 & 12 \\
\hline $\begin{array}{l}\text { Grey-headed Puff-back } \\
\text { Flycatcher }\end{array}$ & Batis minima & 10.4 & 114.1 & 5 & 0 & 5 \\
\hline Chestnut-capped Flycatcher & Erythrocercus mccalli & 7.0 & 101.6 & 1 & 0 & 1 \\
\hline White-browed Forest Flycatcher & Fraseria cinerascens & 44.3 & 177.3 & 4 & 1 & 5 \\
\hline Fraser's Forest Flycatcher & Fraseria ocreata & 41.8 & 174.8 & 1 & 0 & 1 \\
\hline White-eyed Flycatcher & Muscicapa caerulescens & 14.0 & 127.0 & 18 & 0 & 18 \\
\hline Blissett's Wattle-eye & Platysteira blissetti & 9.8 & 88.9 & 2 & 0 & 2 \\
\hline Chestnut Wattle-eye & Platysteira castanea & 12.0 & 101.6 & 1 & 0 & 1 \\
\hline White-spotted Wattle-eye & Platysteira tonsa & 8.8 & 101.5 & 4 & 0 & 4 \\
\hline Red-bellied Paradise Flycatcher & Terpsiphone rifiventer & 15.0 & 114.3 & 4 & 1 & 5 \\
\hline \multirow{2}{*}{$\begin{array}{l}\text { Blue-headed Crested } \\
\text { Flycatcher }\end{array}$} & Trochocercus nitens & 12.1 & 152.4 & 1 & 0 & 1 \\
\hline & Nectarinidae & & & & & \\
\hline Collared Sunbird & Anthreptes collaris & 8.1 & 101.6 & 7 & 0 & 7 \\
\hline Grey-chinned Sunbird & Anthreptes rectirostris & 8.0 & 153.3 & 6 & 0 & 6 \\
\hline Blue-throated Brown Sunbird & Nectarinia cyanolaema & 8.0 & 53.4 & 10 & 1 & 11 \\
\hline Olive Sunbird & Nectarinia olivacea & 9.2 & 139.7 & 13 & 1 & 14 \\
\hline Superb Sunbird & $\begin{array}{l}\text { Nectarinia superba } \\
\text { Ploceidae }\end{array}$ & 9.8 & 161.2 & 14 & 0 & 14 \\
\hline Blue-billed Malimbe & Malimbus nitens & 33.0 & 190.6 & 13 & 5 & 18 \\
\hline Red-headed Malimbe & Malimbus rubricollis & 28.6 & 183.4 & 6 & 6 & 12 \\
\hline Maxwell's Black Weaver & Ploceus albinucha & 20.6 & 165.4 & 1 & 0 & 1 \\
\hline Spectacled Weaver & Ploceus nigricollis & 20.6 & 166.2 & 7 & 5 & 12 \\
\hline Yellow-mantled Weaver & $\begin{array}{l}\text { Ploceus tricolor } \\
\text { Estrildinidae }\end{array}$ & 24.2 & 175.8 & 3 & 0 & 3 \\
\hline Chestnut-breasted Negro-finch & Nigrita bicolor & 11.0 & 114.3 & 13 & 2 & 15 \\
\hline White-breasted Negro-finch & Nigrita fusconota & 9.6 & 101.6 & 8 & 1 & 9 \\
\hline Pale-fronted Negro-finch & Nigrita luteifrons & 9.5 & 114.3 & 12 & 0 & 12 \\
\hline \multirow{3}{*}{ Blue-billed Weaver } & Spermophaga haematina & 18.0 & 152.4 & 6 & 1 & 7 \\
\hline & Total number of captures & & & 584 & 386 & 970 \\
\hline & Total number of species & & & 93 & 73 & - \\
\hline
\end{tabular}
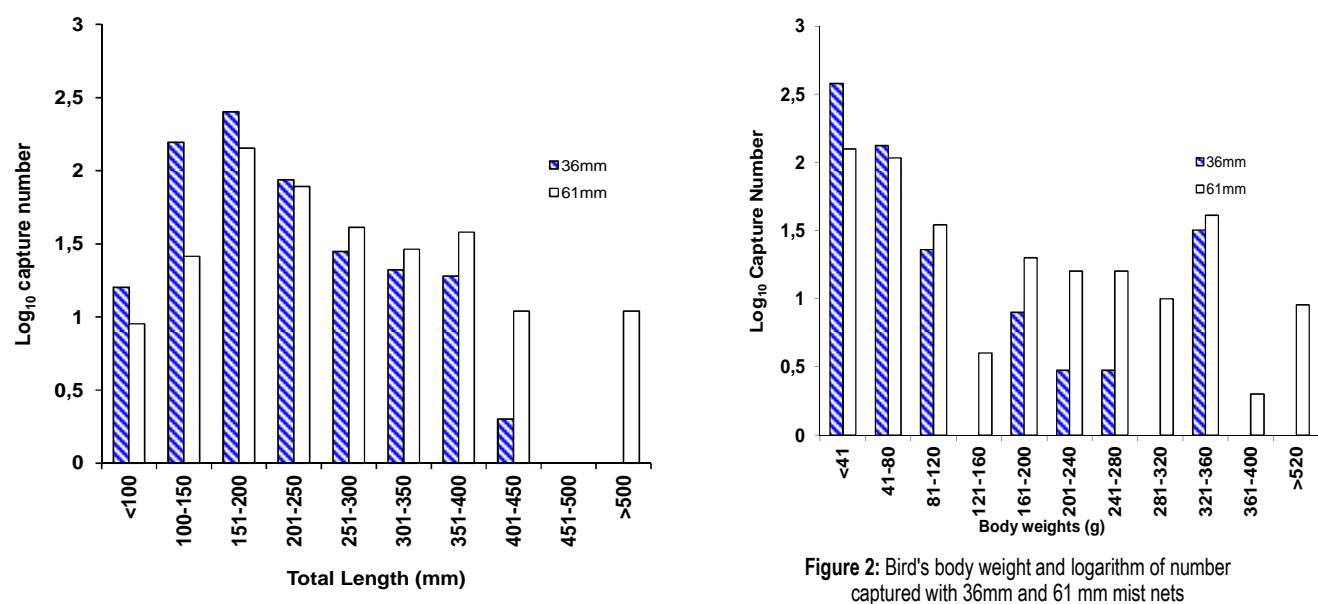

Figure 2: Bird's body weight and logarithm of number captured with $36 \mathrm{~mm}$ and $61 \mathrm{~mm}$ mist nets

Figure 1: Bird's total length $(\mathrm{mm})$ and logarithm of number captured with $36 \mathrm{~mm}$ and $61 \mathrm{~mm}$ mist nets 
Table 2: Mean number of observed but not mist-netted birds in Shasha Forest Reserve, lle-Ife.

\begin{tabular}{|c|c|c|}
\hline Common Name & Family/Species & Mean Number \\
\hline & Accipitridae & \\
\hline West African River Eagle & Haliaetus vocifer & 7 \\
\hline Bat Hawk & Macheiramphus alcinus & 18 \\
\hline Black kite & Milvus migrans & 11 \\
\hline & Bucerotidae & \\
\hline Brown-cheeked Hornbill & Bycanistes cylindricus & 3 \\
\hline Piping hornbill & Bycanistes fistulator & 2 \\
\hline Black-casqued Hornbill & $\begin{array}{l}\text { Ceratogymna atrata } \\
\text { Capitonidae }\end{array}$ & 3 \\
\hline Tooth-billed barbet & Lybius bidentatus & 9 \\
\hline Hairy-breasted toothbill & $\begin{array}{l}\text { Lybius hirsutus } \\
\text { Picidae }\end{array}$ & 16 \\
\hline Green-backed Woodpecker & $\begin{array}{l}\text { Campethera cailliautii } \\
\text { Pycnonotidae }\end{array}$ & 23 \\
\hline Simple leaf-love & Chlorocichla simplex & 6 \\
\hline Spotted Bulbul & $\begin{array}{l}\text { Ixonotus guttatus } \\
\text { Muscicapidae }\end{array}$ & 2 \\
\hline Dusky Blue Flycatcher & Muscicapa comitata & 3 \\
\hline
\end{tabular}

\section{DISCUSSION}

The demonstration that mist nets are an effective means of assessing population of birds has encouraged their widespread introduction to various parts of the world. However, there are major operational difficulties in ensuring that mistnets with appropriate mesh sizes are used.

O'Oconnor [12] suggested that mist nets do not sample juvenile and adult equally in the same geographical area. This discrepancy was confirmed by Nur and Geuper [13] who stated that among Wren tits, the physical area sampled by nets varied among age classes. The results presented here imply that the area sampled by mist nets may differ for adults of different species, but in all cases the efficiency with which the nets monitor local population trends decreases with distance from nets. Mist-netting can give reliable information on trends in capture rates of local population for several species on species by species basis.

It is interesting to note that body length and weight of birds are related to mesh size capture rates but Jenni et al., [6] indicate that body weight and cranium size are more useful measures than total body length alone for determining capture rates. So a combination of total body length and weight would provide a more meaningful capture rates. Hence, there is a dividing line between relative efficiencies of the two mesh types. There is no overlap and no group of birds in which, statistically significant, equal numbers were caught in both mesh sizes. It was expected, of course, that the smaller mesh would catch more smaller birds, and vice-versa, but the statistical analysis showed what a surprising difference mesh size actually made. The effects of the two types of nets was also seen in the total catch of $584(60.2 \%)$ for the $36 \mathrm{~mm}$ mesh, versus $386(39.8 \%)$ for $61 \mathrm{~mm}$ for mesh. This difference is also most statistically significant $(P<0.01)$. The statements of Jenni et al. [6] and Remsen and Good [5] that behavioural differences leading to differences in capture probability are more pronounced when comparing different species than when comparing within species was also displayed in this study.

Although, this study was restricted to mist-netted birds, there were other observed birds not netted. The reason for a species not being caught may be due to the fact that its foraging activity was largely confined to the forest canopy and rarely descended to the level of the nets, which reached 2.6 metres above the ground. Some predominantly canopy species were nevertheless caught, but were netted much less often in relation to their abundance than were species that regularly used the understory. A few other bird species could also not be caught because they were either large enough to break out of or avoid entanglement in the nets or aerial in activity like that Bat Hawk (Macheiramphus alcinus), Black Kite (Milvus migrans) and West African River Eagle (Haliaetus vocifer). This observation buttressed the reports of Diamond [14] in New Guinea and Terborgh and Weske [15] in Peru who 
labeled some birds as "uncatchable" because of being confined to the canopy.

If the two mist nets are deployed simultaneously and systematically, and if the results are interpreted on a species by species basis, this will provide an improvement of abundance estimates for some larger species. By using only $36 \mathrm{~mm}$ mesh mist nets there may be an increase in the capture rate, particularly when there is a greater density of small birds as observed in tropical forest understories. For bigger and heavier bird species, the $61 \mathrm{~mm}$ mesh would substantially improve the capture rate. The result has equally shown that banders who wish to catch the broadest possible range of bird species should use several mesh sizes in their net lanes. The proportionate numbers of each mesh will be determined by the size composition of the particular fauna. Researchers interested in general population surveys would equally use a variety of net types but those concentrating on single species should use the most efficient mesh size for that species from already existing data base. It is hoped that similar studies would be conducted with other mesh sizes so that banders and field Ornithologists would have information available on the comparative efficiencies of birds caught in 36 and $61 \mathrm{~mm}$ mesh nets.

\section{ACKNOWLEDGMENT}

I wish to thank Osun State Ministry of Agriculture and National Resources, Nigeria for their support and approval of the study site, and particularly the Forest Guards (Rangers) who generously agreed to participate in this study. I am grateful to Mr. B. Faloba of Natural History Museum who placed some materials at my disposal and Mr. U. Amakulo of the Institute of Ecology and Environmental Management, O.A.U., who took part in the field work.

\section{REFERENCES}

1. Macarthur R.H., Macarthur AT. 1974. On the use of mist nets for population studies of birds. Proceedings National Academy of Science 7(8): 3230-3233.

2. Ralph C., Geupel G.R., Pyle P., Martin T.E. and Desante D.F. 1993. Handbook of field methods for monitoring landbirds. USDA Forest Service General Technical Report PSW-GTR-144. 41p.

3. Poulin B., Lefebvre G. and Pilard P. 2000. Quantifying the breeding assemblage of reedbed passerines with mist-net and pointcount surveys. Journal of Field Ornithology 7(3): 443-454.

4. Rappole J.H., Winker K. and Powell G.V.N. 1998. Migratory bird habitat use in Southern Mexico: Mist nets versus point counts. Journal of Field Ornithology 69(4): 635-643.

5. Remsen J.V. and Good D.A. 1996. Misuse of data from mist-net captures to assess relative abundance in bird populations. Auk 113: 381398.

6. Jenni L., Leuenberger M. and Rampazzi $F$. 1996. Capture efficiency of mist nets with comments on their role in the assessment of Passerine habitat use. Journal of Field Ornithology 67(2): 263-274.

7. Young B.E., Derosier D. and Powell G.V.N. 1998. Diversity and conservation of understory birds in Tilaran Mountains, Costa Rica. Auk 115(4): 998-1016.

8. Silkey M., Nur N. and Geupel G.R. 1999. The use of mist-net capture rates to monitor annual variation in abundance: a validation study. Condor 101(2): 288-298.

9. Stratford J.A. and Stouffer P.C. 1999. Local extinctions of terrestrial insectivorous birds in a fragmented landscape near Manaus, Brazil. Conservation Biology 13(6): 1416-1423.

10. Fowler J. and Cohen L. 1995. Statistics for ornithologists. British Trust for Ornithology, Norwich.

11. Serle W., Morel G.J. and Hartwig W. 1984. A Field Guide to the Birds of West Africa. William Collins Sons \& Co. Ltd. London. $351 \mathrm{pp}$.

12. O'oconnor J.R. 1992. The analysis of geographic scale and population processes in bird population monitoring data. In McKenzie D.H., Hyatt D.E. \& McDonald (eds.). Ecological Indicators. Elsevier, London. p. 929-960.

13. Nur N. and Geuper G.R. 1993. Validating the use of constant effort mist-netting to monitor avian populations. Report of the Point Reyes Bird Observatory to the US Dept. of Interior, Fish and Wildlife Service of Migratory Bird Management Stinson Beach. CA.

14. Diamond J.M. 1972. Avifauna of the Eastern Highlands of New Guinea (Nuttal Ornithological Club, Cambridge, Mass.)

15. Terborgh J.W. and Weske J.S. 1969. Colonization of secondary habitat by Peruvian birds. Ecology 50(5): 765-782. 